\title{
Screening and evaluation of selenium-rich maize cultivars in Ziyang area
}

\author{
Yan $\mathrm{Li}^{1,2}$, Jianwu Yue ${ }^{3}$, Zhibin Duan ${ }^{3}$, Shuangquan $\mathrm{Wu}^{3}$, Bo Li ${ }^{1}$, Chao Sun ${ }^{1}$, Jiakun Dai ${ }^{2 *}$, and Bingde Dou ${ }^{2 *}$ \\ ${ }^{1}$ Microbiology Institute of Shaanxi, Xi'an 710043, Shaanxi, China \\ ${ }^{2}$ Bio-Agriculture Institute of Shaanxi, Xi'an 710043, Shaanxi, China \\ ${ }^{3}$ Agriculture and Rural Bureau of Ziyang, Ankang 725300, Shaanxi, China
}

\begin{abstract}
To study selenium (Se)-enriched characteristics of maize in Ziyang area and to select cultivars, six cultivars were planted on three different soils with high-Se, middle-Se and low-Se. The Se content of grain, stalk \& leaf and cob were tested separately, and the relationship between Se content in soil and the capability of Se-enrichment in different organs of six cultivars were analyzed. The result showed that the Se content of maize organs is positively correlated with the Se in soil. Meanwhile, on the soil with different Se content, the trends of Se-enriching capacity in different organs of maize cultivars were as follows: stalk \& leaf $>$ grain $>$ cob. Further comparison of the ratio of the Se content of various organs to the Se content of soil showed that, the maize planted on the middle-Se soil has the highest capability of Se-enrichment. Considering the biomass, the accumulation of Se in the grains of sweet maize Shaan K512 and waxy maize Shaan K818 were relatively higher. This study has implications for Se-rich maize and comprehensive utilization of Se in Ziyang.
\end{abstract}

efficiently.

\section{Introduction}

Selenium (Se) is an essential micronutrient for human health. There are more than 20 selenoproteins and selenases participate in normal metabolism ${ }^{[1]}$. The deficiency of Se is closely related to many diseases, such as Keshan disease, white muscle disease, cardiovascular disease and cancer [2]. However, the distribution of selenium in the world's soil is extremely uneven. The report of World Health Organization has pointed out that at least more than 40 countries and regions lack Se in soil [3]. In China, $72 \%$ of the soil is in Se-deficiency. The selenium content in the low-Se area is less than 0.125 $\mathrm{mg} / \mathrm{kg}$, which is much lower than the international average soil Se content $(0.4 \mathrm{mg} / \mathrm{kg})^{[4]}$. As Se-rich agricultural products are beneficial to human health, people increase the Se content by foliar application, seed soaking and seed dressing with selenium fertilizer ${ }^{[5,6]}$, but there are few reports about natural selenium enrichment in agriculture.

Ziyang is a well-known natural selenium-rich area in Shaanxi Province of China, which is famous for the selenium-rich tea ${ }^{[7]}$. Shuang'an town is the selenium-rich core area of Ziyang, and it is also a very poverty-stricken area in China. Therefore, the screening and promotion of selenium-rich agricultural products is important to the local economy. Recent research shows that maize has significant Se-enrichment effect ${ }^{[8]}$. In order to screen out the maize cultivars with high Se-enrichment effect, six maize cultivars were detected in this study, which would help the local utilization of natural selenium resources

\section{Materials and Methods}

\subsection{Experimental design}

Three fields with different selenium contents were selected for the experiment, in Shuang'an Town, Ziyang County, Shaanxi Province $\left(32.52^{\circ} \mathrm{N}, 108.53^{\circ} \mathrm{E}\right)$. The area belongs to the subtropical humid monsoon climate zone, with an average annual temperature of $15.1{ }^{\circ} \mathrm{C}$, total annual precipitation of $1066 \mathrm{~mm}$, and frost-free period of 268 days. The test areas were flat or gentle slope with about $1000 \mathrm{~m}^{2}$. The testing maize included five cultivars provided by Bio-Agriculture Institute of Shaanxi: sweet corn (Shaan K512 and Shaan K2912), colored waxy corn (Shaan K818), black waxy corn (Shaan K635), white waxy corn (Shaan K928), and one local field maize (Zhengda 12). The trial was carried out from April to July in 2019. All cultivars are planted in the same distribution on three fields of different Se content, under the same field management and unified water and fertilizer control. The planting density of each cultivar was $4.5 \times 10^{4}$ plants $/ \mathrm{hm}^{2}$.

\subsection{Material collection and testing}

\subsubsection{Soil collection and testing}

Five-points sampling and mixed standard method was used to collect $0-20 \mathrm{~cm}$ soil samples below the ground.

"Corresponding authors: djkxa@163.com; doubd@163.com 
According to the agricultural industry standard: determination of total selenium in soil NY/T1104-2006 [9], the total Se contents of the samples were detected.

\subsubsection{Maize sample collection}

The maize samples were collected when they were mature. 20 typical plants in each group were cut randomly, and the whole plant's grain, stalk \& leaf and cob were collected separately. Then, they were put into the electric blast drying oven at $90^{\circ} \mathrm{C}$, and dry at $60^{\circ} \mathrm{C}$ to constant weight.

\subsubsection{Biomass determination}

The collected grain samples were weighed and the biomass was calculated.

\subsubsection{Selenium content determination}

According to the national food safety standard: determination of selenium in food GB 5009.93-2017 [10], the Se content in grain, stalk \& leaf and cobs was detected respectively.

Se-enrichment capacity is the ability of crops to enrich Se from soil.

Se-enrichment capacity $=$ Se content in crop organs / Se content in soil.

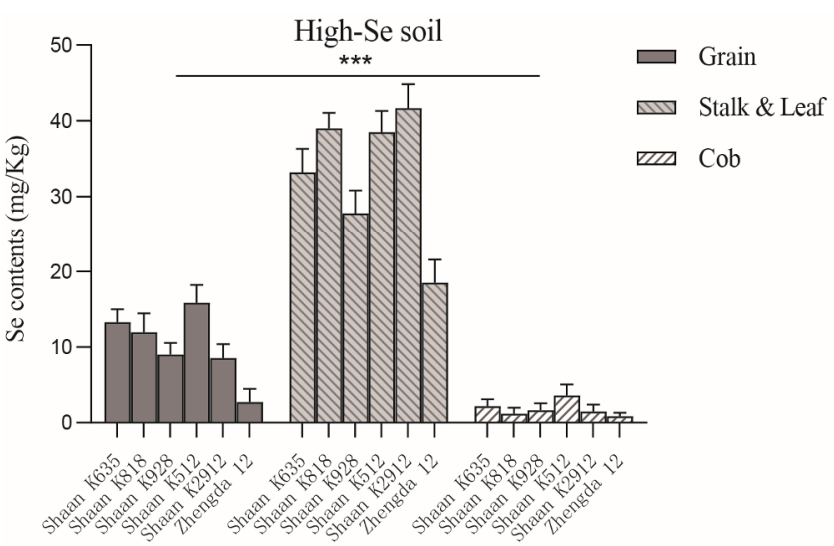

Se-accumulation is the total amount of Se accumulated by crops from soil.

Se-accumulation $=$ Se content $\times$ crop biomass.

\subsection{Data statistics}

Microsoft Excel 2010 was used to process the data, SPSS 26.0 was used for cultivars analysis, and least significant differences (LSD) method was used for multiple comparisons $(\mathrm{P}<0.05)$.

\section{Results and Discussion}

\subsection{Analysis of Se content in different cultivars}

According to the detection of Se content in soil, three experimental fields were selected, which were high-Se soil $(5.49 \mathrm{mg} / \mathrm{kg})$, middle-Se soil $(3.02 \mathrm{mg} / \mathrm{kg})$ and lowSe soil $(0.26 \mathrm{mg} / \mathrm{kg})$.

The results of the Se-distribution showed that maize have different Se contents when planted in different soils. The trend of Se content of maize in different soils was: high-Se soil $>$ middle-Se soil $>$ low-Se soil. Meanwhile, the trend of Se-enrichment effects of organs was: stalk \& leaf $>$ grain $>$ cob (Fig. 1).

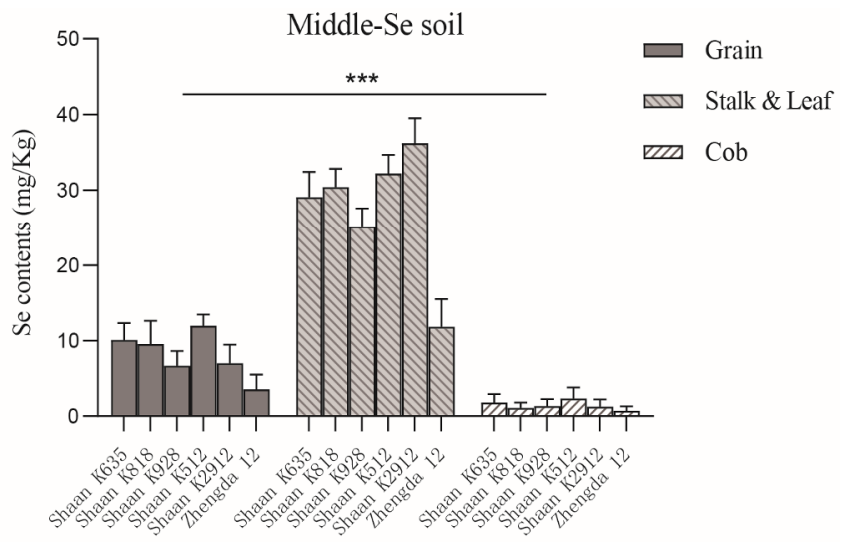

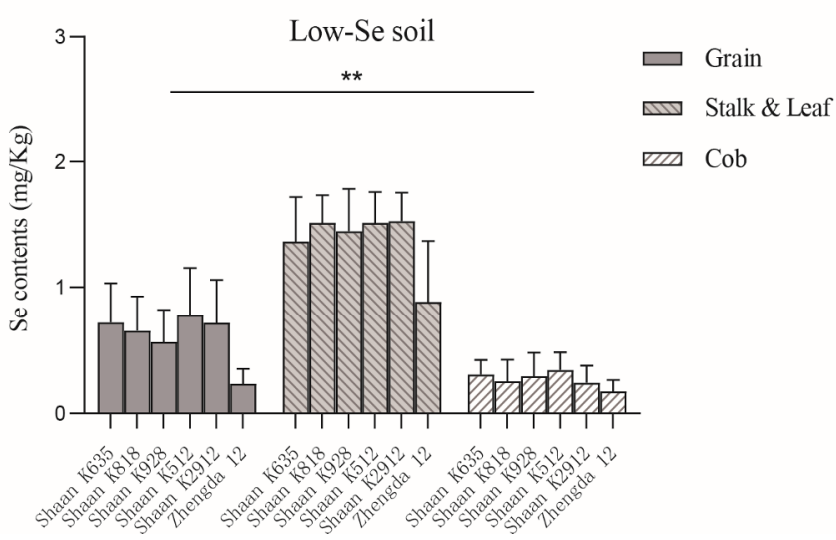

Fig.1. Distribution of Se in various organs of maize

\subsection{Analysis of Se-enrichment capacity}

The ratio of Se-content in maize organs to Se-content in soil can reflect the Se-enrichment capacity of organs with different varieties. The organ samples of maize planted on soil with different Se concentrations were numbered, as shown in Table 1.

The results showed that in the soil with different $\mathrm{Se}$ 
content, the Se-enrichment capacity of stalk \& leaf was the highest, followed by the grain, and the cob was the weakest, which was especially obvious in the middle-Se soil. The Se-enrichment capacity of grain was 2-3 times, and the highest (3.96 times) appeared in Shaan K512 (No.
22) planted in middle-Se soil. The Se-enrichment capacity of stalk \& leaf was generally higher than that of grain. And the highest was Shaan K2912 (No. 29) planted in middleSe soil, whose Se-enrichment capacity could reach 12.01 times (Fig. 2).

Table 1. Sample number of each organ of maize planted on soil with different Se concentration

\begin{tabular}{cccccccccc}
\hline Cultivar & \multicolumn{3}{c}{ High-Se soil } & \multicolumn{3}{c}{ Middle-Se soil } & \multicolumn{3}{c}{ Low-Se soil } \\
\cline { 2 - 9 } & Grain & Stalk \& Leaf & Cob & Grain & Stalk \& Leaf & Cob & Grain & Stalk \& Leaf & Cob \\
\hline Shaan K635 & 1 & 7 & 13 & 19 & 25 & 31 & 37 & 43 & 49 \\
Shaan K818 & 2 & 8 & 14 & 20 & 26 & 32 & 38 & 44 & 50 \\
Shaan K928 & 3 & 9 & 15 & 21 & 27 & 33 & 39 & 45 & 51 \\
Shaan K512 & 4 & 10 & 16 & 22 & 28 & 34 & 40 & 46 & 52 \\
Shaan K2912 & 5 & 11 & 17 & 23 & 29 & 35 & 41 & 47 & 53 \\
Zhengda 12 & 6 & 12 & 18 & 24 & 30 & 36 & 42 & 48 & 54 \\
\hline
\end{tabular}

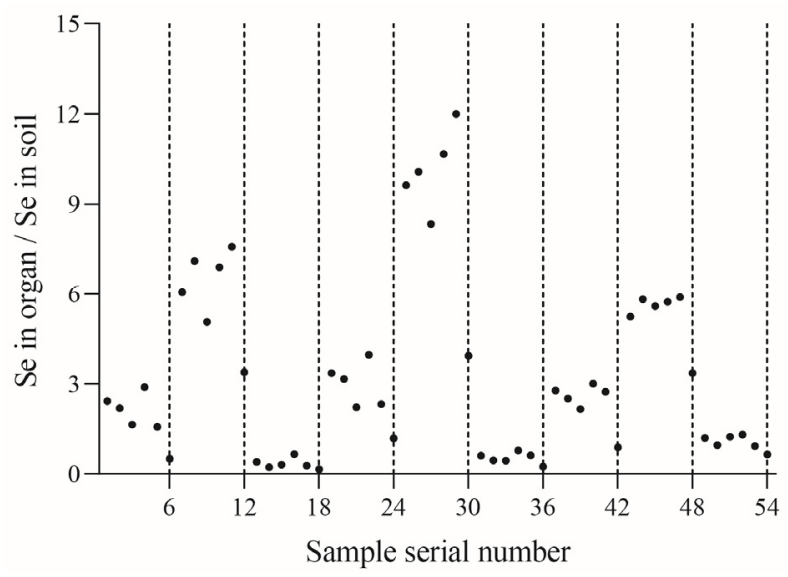

Fig. 2. The ratio of Se-content in organs to Se-content in soil

\subsection{Analysis of se-accumulation in grain}

In the soil with different Se concentration, the analysis result of se-accumulation in grain was shown in Fig. 3 (the area of circle represented the accumulation of Se in grain of each cultivar).

In sweet corn, the Se-accumulation of Shaan K512 was significantly higher than that of Shaan K2912; in waxy corn, the Se-accumulation of Shaan K818 was slightly higher than that of Shaan K635, significantly higher than that of Shaan K928, whether in high-Se or middle-Se soils. There was no significant difference between both sweet and waxy maize cultivars in low-Se soil. Although the local field maize (Zhengda 12) has larger biomass, its selenium content is much lower than the other cultivars, so its Se-accumulation is the lowest.

\section{Conclusions}

The soil selenium content and the maize cultivar could significantly affect the distribution of selenium in organs of sweet and waxy maize, especially in stalk \& leaf and grain. Although the selenium content of corn organs is positively correlated with the soil selenium content, its Seenrichment capacity is not completely correlated with the Se content in soil. The research showed that the grain, stalk \& leaf of maize planted in middle-Se soil have the highest Se-enrichment capacity.

As the main crops in Ziyang area, it was found that there were high Se contents in the grain of sweet and waxy maize, and the by-products (stalk \& leaf) also had high Se content and Se-enrichment capacity. Therefore, in the process of production, the waste stalk \& leaf can be further processed to prepare organic Se products, so as to make more efficient use of Se resources. 

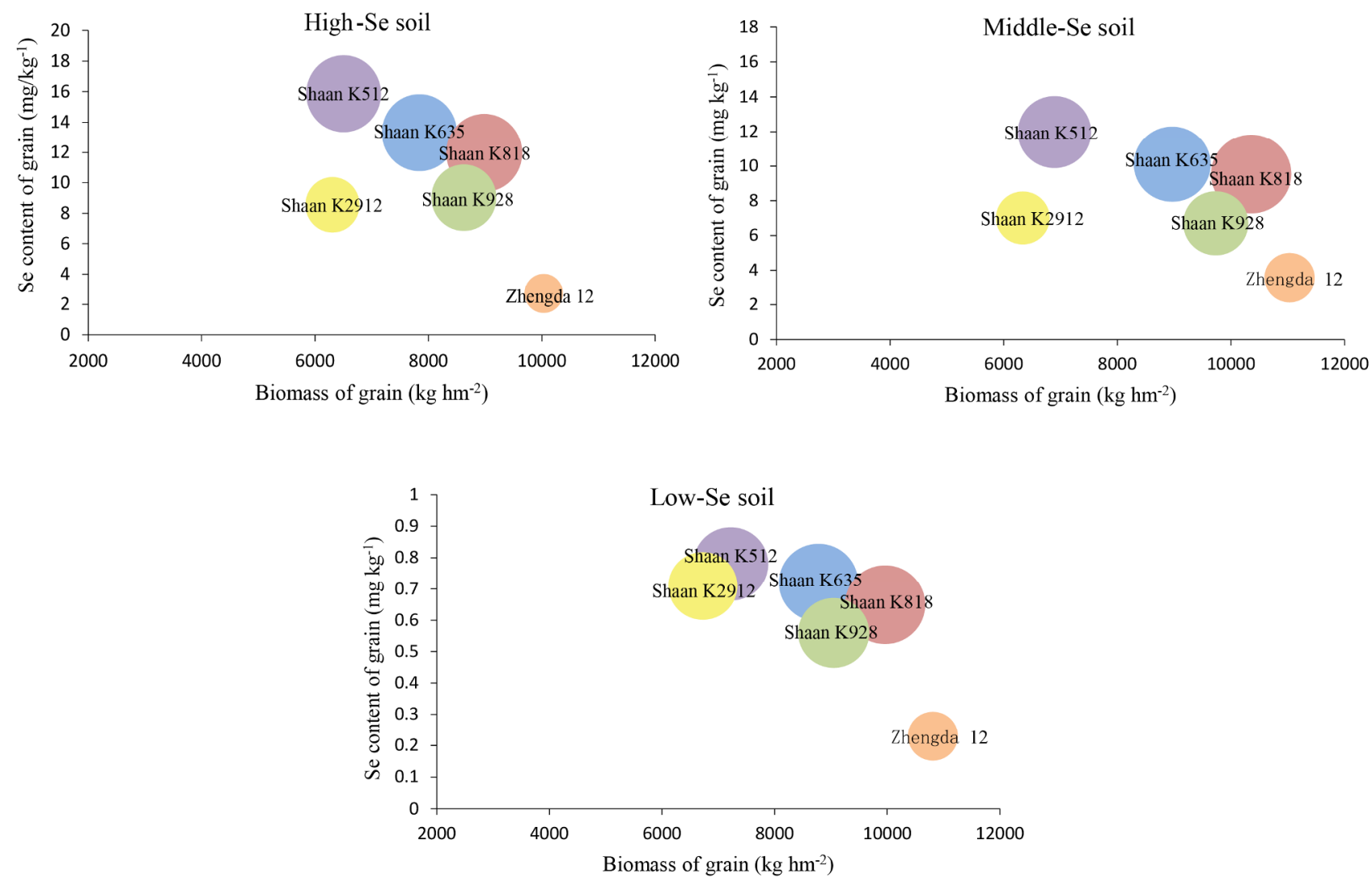

Fig. 3 The Se accumulation in grains of different maize cultivars on soil with different Se content

In summary, we found that compared with the local field maize, the sweet and waxy maize had higher Se utilization in soil. Among the tested cultivars, Shaan K512 and Shaan K818 have stronger Se-accumulation capacity and could be widely planted in Ziyang area.

\section{Acknowledgement}

This work was supported by the Poverty Alleviation Project of Shaanxi Academy of Science (2018K-13), the Key Research and Development Program of Shaanxi Province (2020FP-008) and the Science \& Technology Plan Project of Shaanxi Academy of Science (2020K-16).

\section{References}

1. C.H. Tang, Q.Y. Zhao, K. Zhang, S. Li, Y.C. Qin, J.M. Zhang, Sci Agr Sin, 52, 3122-3133 (2019)

2. Y.F. Yao, F.X. Pei, L. Liang, P.D. Kang, Nutrition, 27, 1095-1100 (2011)

3. Z.C. Xu, H.F. Shao, S. Li, C. Zheng, Pak J Bot, 44, 1563-1568 (2012)

4. Q.T. Dinh, Z.W. Cui, J. Huang, T.A.T. Tran, D. Wang, W.X. Yang, F. Zhou, M.K. Wang, D.S. Yu, D.L. Liang, Environ Int, 112, 294-309 (2018)

5. L. Melanie, C.R. Maryse, B. Philippe, B. Thierry, Food Chem, 182, 128-135 (2015)

6. T.S. Yang, T.Q. Du, H.M. Zhai, Y.H. Li, R. Gong, F.Z. Cui, M. Sun, Z.Q. Gao, J Maize Sci, 28, 117-123
(2020)

7. S.S. Wang, D.L. Liang, D. Wang, W. Wei, D.D. Fu, Z.Q. Lin, Sci Total Environ, 427, 159-164 (2012)

8. Z.Y. Yuan, K.Y. Xu, B. Huang, C.H. Li, W. Xia, B.H. Duan, F. Pan, T.Y. Wang, M. Zhao, J.L. Yan, Resour Environ Eng, 32, 569-575 (2018)

9. Ministry of Agriculture of the People's Republic of China. NY/T1104-2006. Agricultural industry standards: Determination of selenium in soils (2006)

10. Ministry of Health of the People's Republic of China. GB5009.93-2017. National Food Safety Standard: Determination of Selenium in Foods (2017) 